\title{
Multiple Myeloma Treatments, Outcomes, and Costs of Health Care Resource Utilisation During 2009-2016, Based on Multiple Data Sources from a Hospital District in Finland
}

\author{
Saku Torvinen ${ }^{1,}$, , Ville Vihervaara ${ }^{1}$, Tatu Miettinen ${ }^{2}$, Jari Jokelainen ${ }^{3,4}$, Melissa Bauer ${ }^{1}$, \\ Anu Marttila ${ }^{5}$, Klaus Tamminen ${ }^{1}$, Jonna Salonen ${ }^{5,6}$ \\ ${ }^{1}$ Takeda Oy, Helsinki, Finland \\ ${ }^{2}$ Medaffcon Oy, Espoo, Finland \\ ${ }^{3}$ MedEngine Oy, Helsinki, Finland \\ ${ }^{4}$ Unit of General Practice, Oulu University Hospital, Oulu, Finland \\ ${ }^{5}$ Kymenlaakso Central Hospital, Kotka, Finland \\ ${ }^{6}$ Current Affiliation: Finnish Institute for Health and Welfare, Helsinki, Finland
}

\section{Email address:}

saku.torvinen@takeda.com (S. Torvinen), ville.vihervaara@takeda.com (V. Vihervaara), tatu.miettinen@takeda.com (T. Miettinen), jari.jokelainen@medengine.fi (J. Jokelainen),melissa.bauer@takeda.com (M. Bauer), anu.marttila@kymsote.fi ( $\Lambda$. Marttila), klaus.tamminen@takeda.com (K. Tamminen), jonna.salonen@thl.fi (J. Salonen)

${ }^{*}$ Corresponding author

\section{To cite this article:}

Saku Torvinen, Ville Vihervaara, Tatu Miettinen, Jari Jokelainen, Melissa Bauer, Anu Marttila, Klaus Tamminen, Jonna Salonen. Multiple Myeloma Treatments, Outcomes, and Costs of Health Care Resource Utilisation During 2009-2016, Based on Multiple Data Sources from a Hospital District in Finland. International Journal of Clinical and Experimental Medical Sciences. Vol. 7, No. 1, 2021, pp. 21-30. doi: $10.11648 /$ j.ijcems.20210701.14

Received: December 31, 2020; Accepted: January 11, 2021; Published: January 28, 2021

\begin{abstract}
Multiple myeloma (MM) is one of the most prevalent hematologic cancers. Treatments of MM have been improved by availability of novel therapies but require regular hospital visits and intense patient follow-up. In this real-world study, patient characteristics, first four treatment lines ( $1 \mathrm{~L}-4 \mathrm{~L})$, and associated outcomes and costs were assessed among adults treated for active MM during 2009-2016 at Kymenlaakso Central Hospital, Kymsote hospital district, Finland. In addition, patient burden and travel costs were determined for the patients treated during 2015-2016. Ninety-seven patients fulfilled the inclusion criteria. Data were retrospectively collected from hospital's database, medical charts, and from healthcare professionals. Treatment lines and responses were defined according to the general recommendations. The median age at diagnosis was 70.1 years. The median overall survival was 68 months. Proteasome inhibitors (PI) or immunomodulatory drugs (IM) were the most common regimen types while the utilisation of a more novel approach, the simultaneous use of PI and IM, was low across first four treatment lines. Overall response rate was $72-74 \%$ for $1 \mathrm{~L}-2 \mathrm{~L}$ and $50-56 \%$ for $3 \mathrm{~L}-4 \mathrm{~L}$. Drug costs represented the greatest proportion of total healthcare costs and increased in the later treatment lines. Patients receiving infusion treatments had specialised health care visits twice as much the patients treated with oral treatments. Furthermore, travel costs related to infusion treatments were three to four times more compared to the respective costs for oral treatments. Increasing drug costs but poorer treatment outcomes in later treatment lines underline a need for more efficient and better tolerated treatment options. This study demonstrates that oral treatments may indeed reduce patient and hospital resource burden and thus, should be considered in future health economic evaluations in Finland.
\end{abstract}

Keywords: Healthcare Costs, Line of Treatment, Multiple Myeloma, Oral Treatment, Survival, Treatment Outcome 


\section{Introduction}

Multiple myeloma (MM, International Classification of Diseases 10th Revision, ICD-10, diagnosis code C90.0) is a $\mathrm{B}$ cell/plasma cell malignancy resulting from a complex series of mutations and clonal selection events that lead to the clonally heterogenous neoplasm of $\mathrm{B}$ cells in the bone marrow and overproduction of monoclonal immunoglobulins often causing organ/tissue damage $[1,2]$. At symptomatic stage, MM is clinically characterised by several symptoms, including bone pain and lesions, myelosuppression and infections, kidney failure, anaemia, and neuropathy [1,3].

$\mathrm{MM}$ is one of the most prevalent hematologic cancers, and the most common plasma cell cancer type [4-6]. It is more often diagnosed in men and in elderly patients with a median age of diagnosis being $65-70$ years [4, 6-8]. The incidence of MM has increased globally [9], and this trend is expected to continue in parallel with the increasing proportion of elderly patients [10]. In 2016, the worldwide age-adjusted incidence estimate of MM was 2.1 per 100000 [9]. According to the available national literature and Finnish Cancer Registry data on myeloma and plasma cell cancers, around 300-400 new myeloma cases are diagnosed annually in Finland (1\% of all cancers) with the unadjusted incidence being 5-7/100 000/year (the age-adjusted incidence around 3/100 000/year) $[6,11-13]$.

MM and its complications involve treatment combinations that are often complex, regular hospital visits, and intense patient follow-up [13-17]. The development of autologous stem cell transplantation (ASCT) followed by immunomodulatory drugs (IM), proteasome inhibitors (PI), and more recently, monoclonal antibodies has improved the treatment of MM during the past two decades [18-22]. In accordance, the guidelines have been revised several times [14, 16, 17]. Patient's clinical condition and age are considered throughout the therapy lines. In Finland, patients in good health and aged under 70-75 years are treated with induction treatment in combination with novel agents, glucocorticoids and often chemotherapy followed by ASCT and usually maintenance therapy [14]. Fit patients aged up to 85 years ineligible for ASCT are treated with novel therapy and others with conventional therapy [14]. A spectrum of options is available for all first-line patients and the complexity further increases along subsequent line of treatments [14]. Despite the introduction of new intravenous and oral drugs with longer progression-free and overall survival times [18], myeloma remains incurable. According to the most recent estimates the 5-year survival rate of MM in the United States (US) is 53.9\% (data from 2010-2016; [4]). In Finland, $41.0 \%$ of all plasma cell cancer (ICD-10 diagnosis code C90) patients survive by five years after diagnosis (data from 2015-2017; [6]).

Utilisation of real-world evidence (RWE) in the assessment of MM treatment patterns, novel agents, subsequent outcomes, the costs of health care resource utilisation (HCRU), and patient burden has increased along the changing landscape of MM treatment and increasing health economic burden [23-27]. The burden of MM and its treatment is not fully known in the Finnish setting. Local health care registers contain routinely saved patient information that could potentially be important for various types of studies but the utilisation of this information for improving quality of patient treatment in general or analysing various treatment-related costs is limited. Recently an RWE study of Finnish MM patients reported on the longer overall survival and time-to-next treatment of patients who received novel agents and ASCT as a first-line therapy compared with patients without ASCT and/or novel therapies [22]. In addition, patient burden and costs related to hospital-administered drugs were assessed across Finland with the results highlighting the role of novel oral medications in reducing MM-related costs and patient burden [28].

In this observational study, real-world data sources were utilised to describe patient characteristics, treatment patterns, and outcomes among adults treated for active MM during 2009-2016 at Kymenlaakso Central Hospital of the federation of municipalities in social and health services in the region of Kymenlaakso in Finland (Kymsote). In addition, the costs associated with HCRU were assessed. Furthermore, MM patient burden in terms of travels to specialised health care visits and associated costs stratified by type of drug administration was also determined in a sub-group of patients treated during 2015-2016.

\section{Materials and Methods}

\subsection{Patient Inclusion Criteria}

Adults with at least one diagnosis code for active MM (ICD-10 diagnosis code C90.0) and who, according to the Kymenlaakso Central hospital's database, received medication for MM during 01 January 2009 through 31 December 2016, inclusive, at Kymenlaakso Central Hospital, Kymsote were eligible for the study. Patients were excluded from the study if any of the following criteria were met: a diagnosis date before 01 January 2009 or after 31 December 2016 (verified from patient charts), allogeneic transplantation for MM, amyloidosis, or plasma cell leukaemia. In addition, patients treated outside of Kymenlaakso Central Hospital were not eligible for this study. The only exception was autologous stem cell transplantation and stem cell harvesting which was performed at any of the five university hospitals of Finland. The rest of the treatment of these patients was performed in Kymenlaakso Central Hospital. Index date was defined as the date of diagnosis which was collected by a nurse or a doctor either from medical charts, from notification to Finnish Cancer Registry, or from specific chemotherapy form. If not found from any of these sources, the index date was the date of diagnostic test result for bone marrow flow cytometry or bone marrow biopsy.

A sub-cohort utilised in the assessment of patient burden and costs related to travels to health care visits included all patients eligible for the study and who had at least one hospital 
visit related to $\mathrm{MM}$ at Kymenlaakso Central Hospital, Kymsote 01 January 2015 through 31 December 2016.

\subsection{Analyses on Baseline Characteristics}

Three data sources were used to collect data: Kymenlaakso Central Hospital's database, Kymenlaakso Central Hospital's medical charts, and interviews with Kymenlaakso Central Hospital's healthcare professionals treating patients diagnosed with active MM. Descriptive analyses included patient characteristics, MM treatment patterns, outcomes, and costs associated with HCRU and travels to the first four lines of treatment.

Risk stratification was done according to the Revised International Staging System (R-ISS) which allows the identification of three distinct prognostic subgroups (R-ISS IIII) [29]. R-ISS is based on serum beta- 2 microglobulin (B2M), albumin and lactate dehydrogenase (LDH) levels, and the presence of high-risk chromosomal abnormalities analysed with fluorescence in situ hybridization (iFISH). R-ISS stage was manually calculated from existing laboratory results. Threshold values for serum albumin and B2M levels recommended by R-ISS were utilised [29]. The local upper threshold value for normal lactate dehydrogenase in adults was $235 \mathrm{U} / 1$ for patients $<70$ years of age at diagnosis, and $255 \mathrm{U} / \mathrm{l}$ for patients $\geq 70$ years of age at diagnosis. Baseline values for albumin, B2M, and $\mathrm{LDH}$ were defined as values closest to the index. However, values not found within three months from diagnosis were not included in the analyses.

\subsection{Treatment Lines and Responses}

Treatment lines were defined according to the recommendations of International Myeloma Workshop Consensus Panel 1 [30]. Treatments were categorised as oral or infusion treatments with infusions including intravenous and subcutaneous treatments given in the hospital. Abbreviations of treatment regimens are shown in Table 1. Treatment responses associated with the first four treatment lines $(1 \mathrm{~L}-4 \mathrm{~L})$ were evaluated using criteria recommended by the International Myeloma Workshops Consensus Panel 1 [30]. Accordingly, the proportion of patients with "complete response" (CR), "very good partial response" (VGPR), "partial response" (PR), "minimal response" (MR) or "stable disease" (SD), and "progressive disease (PD)" were determined.

\subsection{Costs Associated with Health Care Resource Utilisation}

Costs included all myeloma-related events and recordings that can be derived from patient records and medical charts (i.e. drug administration/prescriptions, inpatient stays, outpatient visits, laboratory tests, and imagings). Inpatient stays and outpatient visits to haematological, internal medicine, infectious, traumatological and emergency departments were considered related to MM. Other costs included transfusions and stem cell transplantations. The costs were extracted from hospital price tariffs and national healthcare unit costs report. Off-treatment costs considered all patients whether or not they had a next line treatment during the follow-up. Medications were determined based on the actual visit schedule and prescribed medications as recorded in the patients' medical charts.

\subsection{Travels to Health Care Visits and Associated Costs}

In order to evaluate the impact of oral treatments versus infusions on travel costs and patient burden, the duration of treatments, the number of all MM-related visits, and the distance travelled to specialised health care units and associated travel costs during $1 \mathrm{~L}-4 \mathrm{~L}$ were separately assessed for each type of drug administration. In addition, the working status during the treatments as well as a need for an escort were determined. The duration of treatments and the number of visits were assessed in a full cohort. Remaining data were based on the analysis of the sub-cohort defined above. The distance between patient's home and hospital as well as travelling time were manually calculated using web mapping services by a study nurse. Costs were calculated from reports received from the nurses.

\subsection{Statistical Analyses}

Table 1. Abbreviations of treatment regimens assessed in the study.

\begin{tabular}{|c|c|}
\hline Abbreviation & Drug combination \\
\hline $\mathrm{Bd}$ & bendamustine + dexamethasone \\
\hline DR-PACE & $\begin{array}{l}\text { dexamethasone } \pm \text { Revlimid (lenalidomide) with infusion } \\
\text { of cisplatin, Adriamycine (doxorubicin), } \\
\text { cyclophosphamide, and etoposide }\end{array}$ \\
\hline DT-PACE & $\begin{array}{l}\text { dexamethasone } \pm \text { thalidomide with infusion of cisplatin, } \\
\text { Adriamycin (doxorubicin), cyclophosphamide, and } \\
\text { etoposide }\end{array}$ \\
\hline KRd & carfilzomib + Revlimid (lenalidomide) + dexamethasone \\
\hline MP & melphalan + prednisone \\
\hline MPR & melphalan + prednisone + Revlimid (lenalidomide) \\
\hline MPT & melphalan + prednisone + thalidomide \\
\hline $\mathrm{R}$ & Revlimid (lenalidomide) \\
\hline RAd & $\begin{array}{l}\text { Revlimid (lenalidomide) + Adriamycine (doxorubicin) }+ \\
\text { dexamethasone }\end{array}$ \\
\hline $\mathrm{Rd}$ & Revlimid (lenalidomide) + dexamethasone \\
\hline Single DXM & dexamethasone \\
\hline $\mathrm{Td}$ & thalidomide + dexamethasone \\
\hline VBd and $\mathrm{R}$ & $\begin{array}{l}\text { Velcade (bortezomib) }+ \text { bendamustine }+ \text { dexamethasone, } \\
\text { and for maintenance, Revlimid (lenalidomide) }\end{array}$ \\
\hline $\mathrm{VCd}$ & $\begin{array}{l}\text { Velcade (bortezomib) + cyclophosphamide }+ \\
\text { dexamethasone }\end{array}$ \\
\hline $\mathrm{Vd}$ & Velcade (bortezomib) + dexamethasone \\
\hline $\mathrm{VdB}$ & Velcade (bortezomib) + dexamethasone + bendamustine \\
\hline VMP & Velcade $($ bortezomib) + melphalan + prednisone \\
\hline VMPT & $\begin{array}{l}\text { Velcade }(\text { bortezomib })+\text { melphalan }+ \text { prednisone }+ \\
\text { thalidomide }\end{array}$ \\
\hline VRd & $\begin{array}{l}\text { Velcade (bortezomib) + Revlimid (lenalidomide) + } \\
\text { dexamethasone }\end{array}$ \\
\hline
\end{tabular}

Distributions of continuous variables were expressed as mean \pm standard deviation (SD) or median and interquartile range (IQR), and categorical variables as numbers and percentage of proportions. Distribution of patients in different treatment lines is illustrated with a Sankey graph. Statistical analyses were conducted using R (version 3.3.1, http://www.r-project.org). 


\section{Results}

\subsection{Patient Characteristics}

During 2009-2016, ninety-nine MM patients were treated at Kymenlaakso Central Hospital, Kymsote. The average population size at Kymsote during the study period was 173 693 inhabitants and therefore, the respective calculated unadjusted incidence for patients treated for active multiple myeloma at Kymsote was 7.1/100 000/year.

Ninety-seven MM patients fulfilled the inclusion criteria. Two patients were excluded due to allogeneic transplantation. The median patient follow-up time in this study was 33.6 months (IQR 14.1-53; mean 36.3 months, range 0.3-90.7). The median age at diagnosis was 70.1 years and $54.6 \%$ were males (Table 2). R-ISS data were available for 48 patients
(49.5\%). A majority, 29 patients (60.4\%), represented stage II patients (Table 2). Stage I and III were rather equally represented in the cohort with eight (16.7\%) and $11(22.9 \%)$ patients, respectively (Table 2). Among the 93 patients $(95.9 \%)$ for whom the data on LDH concentration at diagnosis were available, 72 (77.4\%) had normal LDH levels (Table 2). Cytogenetic testing for chromosomal aberrations was performed for 51 patients $(52.6 \%)$. The most common aberration was 1q21, observed in seven patients (13.7\%), while other tested genetic changes were found in only one to two patients (1.9-3.9\%) (Table 2).

Forty-four patients (45.4\%) died during the follow-up while 53 patients $(54.6 \%)$ were alive at the end of the follow-up. The median overall survival from diagnosis was approximately 68 months (Figure 1).

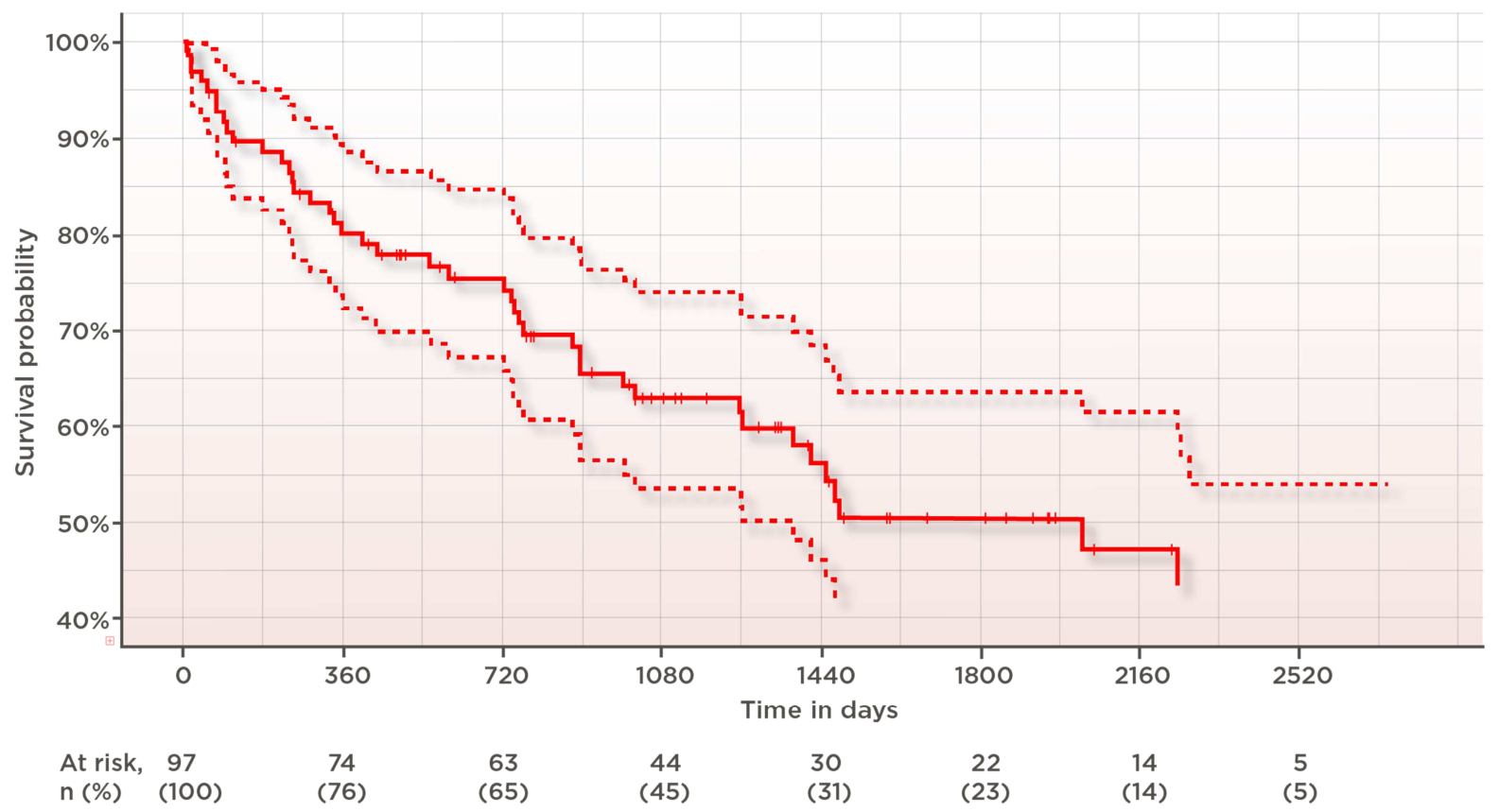

Figure 1. Overall survival from diagnosis of adults treated for active multiple myeloma at Kymenlaakso Central Hospital, Kymsote, Finland, during $2009-2016$. The overall survival was analysed with Kaplan-Meier fits with confidence intervals (dashed lines) and outcome defined as death (event) or end of study (censoring event).

\subsection{Treatment Patterns}

In this study, the first four treatment lines $(1 \mathrm{~L}-4 \mathrm{~L})$ were included in the analyses. From the 97 patients treated in $1 \mathrm{~L}$, 57, 34, and 20 patients continued to $2 \mathrm{~L}, 3 \mathrm{~L}$, and $4 \mathrm{~L}$, respectively (Table 3 ). The number of patients continuing to $5 \mathrm{~L}(\mathrm{n}=10)$ and $6 \mathrm{~L}(\mathrm{n}=4)$ were small and thus, $5 \mathrm{~L}$ and $6 \mathrm{~L}$ were not assessed in this study. Altogether 22 of 97 patients $(22.7 \%)$ received autologous stem cell transplant with 15 $(15.5 \%)$, five $(8.8 \%)$, one $(2.9 \%)$, and one $(5.0 \%)$ treated patients in $1 \mathrm{~L}-4 \mathrm{~L}$, respectively.

The treatment with PI was the most common regimen type in $1 \mathrm{~L}$ with 53 patients treated (54.6\%) (Figure 2). The treatment with IM was the most common regimen in $2 \mathrm{~L}-4 \mathrm{~L}$. Twenty-six (45.6\%), $19(55.9 \%)$ and ten $(50.0 \%)$ patients were treated with IM in $2 \mathrm{~L}-4 \mathrm{~L}$, respectively (Figure 2). The simultaneous use of PI and IM was lowest in all lines of therapy. Only four $(4.1 \%)$, seven $(12.3 \%)$, three $(8.8 \%)$, and three $(15.0 \%)$ patients were treated with PI and IM in $1 \mathrm{~L}-4 \mathrm{~L}$, respectively (Figure 2).

The combination of bortezomib, cyclophosphamide, and dexamethasone (VCd) was the most common drug combination in $1 \mathrm{~L}$ while the combination treatment of lenalidomide and dexamethasone (Rd) was most often used in $2 \mathrm{~L}-4 \mathrm{~L}$. Thirty patients $(30.9 \%)$ were treated with $\mathrm{VCd}$ in $1 \mathrm{~L}$ and $22(38.6 \%), 12(35.3 \%)$, and five $(25.0 \%)$ patients with $\mathrm{Rd}$ in $2 \mathrm{~L}-4 \mathrm{~L}$, respectively (Table 3 ). The mean length of the treatments varied from 144 days in $1 \mathrm{~L}$ to 170 days in $4 \mathrm{~L}$ (Figure 3). 
Table 2. Demographic/clinical characteristics at multiple myeloma diagnosis, among 97 adults treated for active myeloma at Kymenlaakso Central Hospital, Kymsote, Finland, during 2009-2016.

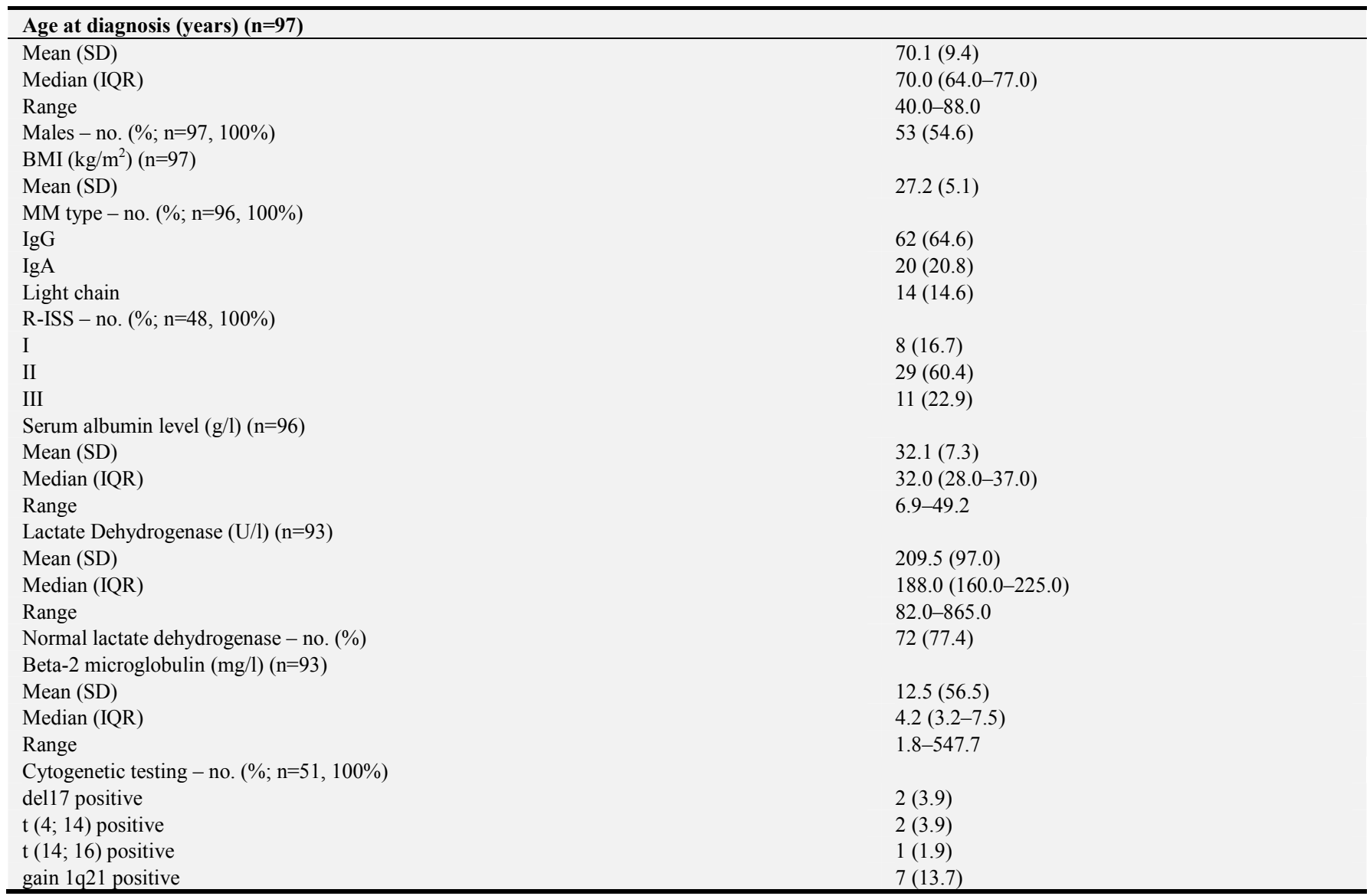

BMI, Body Mass Index; Ig, Immunoglobulin; IQR, Interquartile Range; R-ISS, Revised International Staging System; SD, Standard Deviation.

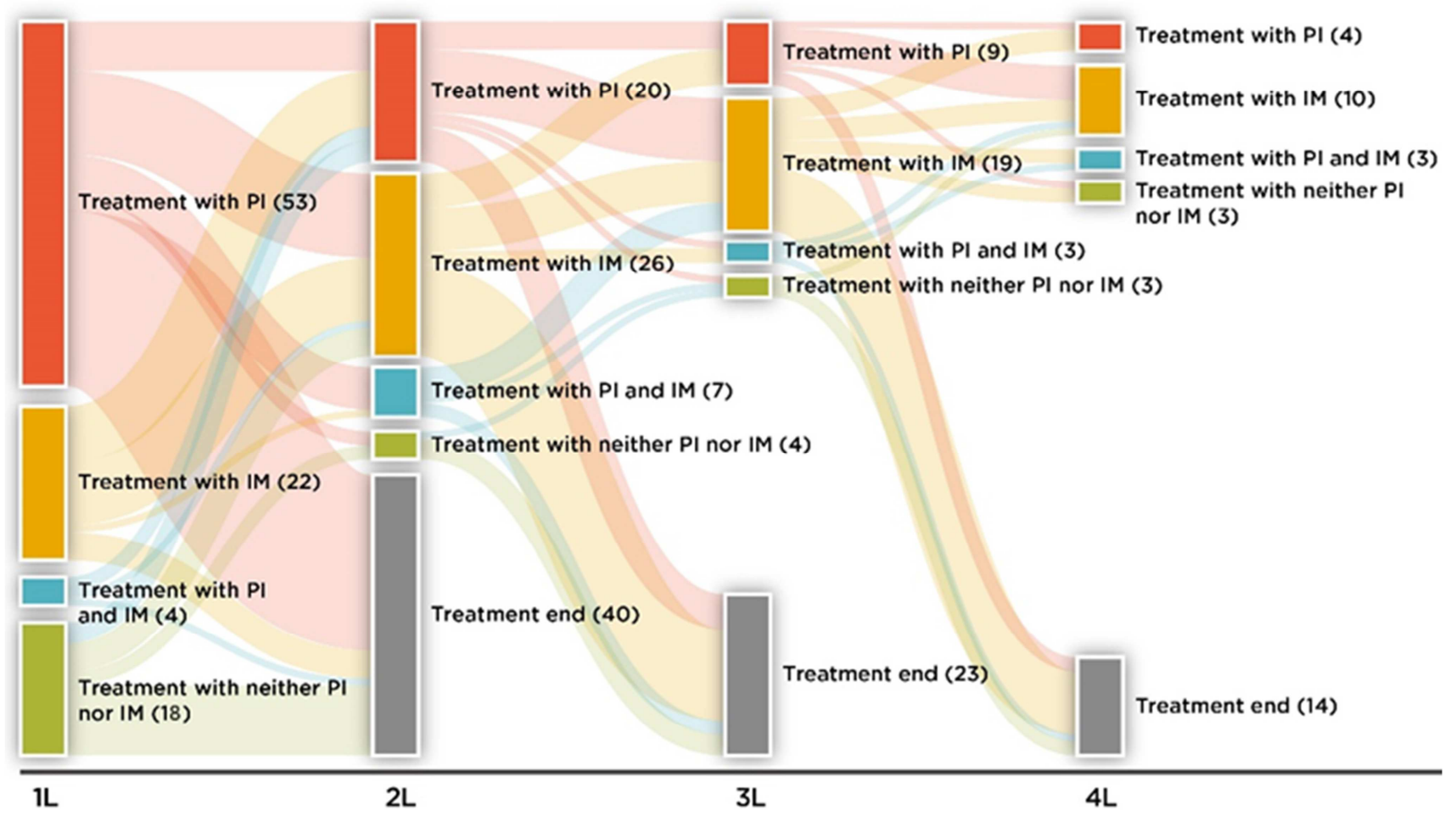

Figure 2. The distribution of patients across the first four treatment lines (1L-4L) stratified by the type of treatment regimen (proteasome inhibitors, PI, and/or immunomodulatory drugs, IM), among 97 adults treated for active multiple myeloma at Kymenlaakso Central Hospital, Kymsote, Finland, during $2009-2016$. 
Table 3. Number of patients treated with distinct regimens in the first four treatment lines (1L-4L), among 97 adults treated for active multiple myeloma at Kymenlaakso Central Hospital, Kymsote, Finland, during 2009-2016.

\begin{tabular}{|c|c|c|c|c|}
\hline Treatment regimen $^{\mathrm{a}}$ & $1 \mathrm{~L}(\mathrm{n}=97,100 \%)$ & $2 \mathrm{~L}(\mathrm{n}=57,100 \%)$ & $3 \mathrm{~L}(\mathrm{n}=34,100 \%)$ & $4 \mathrm{~L}(\mathrm{n}=20,100 \%)$ \\
\hline $\mathrm{Bd}$ & $0(0.0 \%)$ & $0(0.0 \%)$ & $0(0.0 \%)$ & $2(10.0 \%)$ \\
\hline DR-PACE & $0(0.0 \%)$ & $1(1.8 \%)$ & $1(2.9 \%)$ & $0(0.0 \%)$ \\
\hline DT-PACE & $0(0.0 \%)$ & $0(0.0 \%)$ & $1(2.9 \%)$ & $0(0.0 \%)$ \\
\hline KRd & $0(0.0 \%)$ & $0(0.0 \%)$ & $1(2.9 \%)$ & $1(5.0 \%)$ \\
\hline MP & $16(16.5 \%)$ & $2(3.5 \%)$ & $1(2.9 \%)$ & $0(0.0 \%)$ \\
\hline MPR & $0(0.0 \%)$ & $0(0.0 \%)$ & $1(2.9 \%)$ & $0(0.0 \%)$ \\
\hline MPT & $11(11.3 \%)$ & $1(1.8 \%)$ & $1(2.9 \%)$ & $0(0.0 \%)$ \\
\hline $\mathrm{R}$ & $0(0.0 \%)$ & $1(1.8 \%)$ & $2(5.9 \%)$ & $3(15.0 \%)$ \\
\hline RAd & $0(0.0 \%)$ & $0(0.0 \%)$ & $1(2.9 \%)$ & $2(10.0 \%)$ \\
\hline $\mathrm{Rd}$ & $0(0.0 \%)$ & $22(38.6 \%)$ & $12(35.3 \%)$ & $5(25.0 \%)$ \\
\hline Single DXM & $2(2.1 \%)$ & $2(3.5 \%)$ & $2(5.9 \%)$ & $1(5.0 \%)$ \\
\hline $\mathrm{Td}$ & $11(11.3 \%)$ & $1(1.8 \%)$ & $0(0.0 \%)$ & $0(0.0 \%)$ \\
\hline VBd and R maintenance & $0(0.0 \%)$ & $1(1.8 \%)$ & $0(0.0 \%)$ & $0(0.0 \%)$ \\
\hline $\mathrm{VCd}$ & $30(30.9 \%)$ & $6(10.5 \%)$ & $3(8.8 \%)$ & $0(0.0 \%)$ \\
\hline $\mathrm{Vd}$ & $14(14.4 \%)$ & $8(14.0 \%)$ & $1(2.9 \%)$ & $1(5.0 \%)$ \\
\hline $\mathrm{VdB}$ & $0(0.0 \%)$ & $0(0.0 \%)$ & $0(0.0 \%)$ & $1(5.0 \%)$ \\
\hline VMP & $9(9.3 \%)$ & $6(10.5 \%)$ & $5(14.7 \%)$ & $2(10.0 \%)$ \\
\hline VMPT & $1(1.0 \%)$ & $0(0.0 \%)$ & $0(0.0 \%)$ & $0(0.0 \%)$ \\
\hline VRd & $3(3.1 \%)$ & $6(10.5 \%)$ & $2(5.9 \%)$ & $2(10.0 \%)$ \\
\hline
\end{tabular}

${ }^{\text {a }}$ Abbreviations provided in the Table 1. 1L, First Treatment Line; 2L, Second Treatment Line; etc.

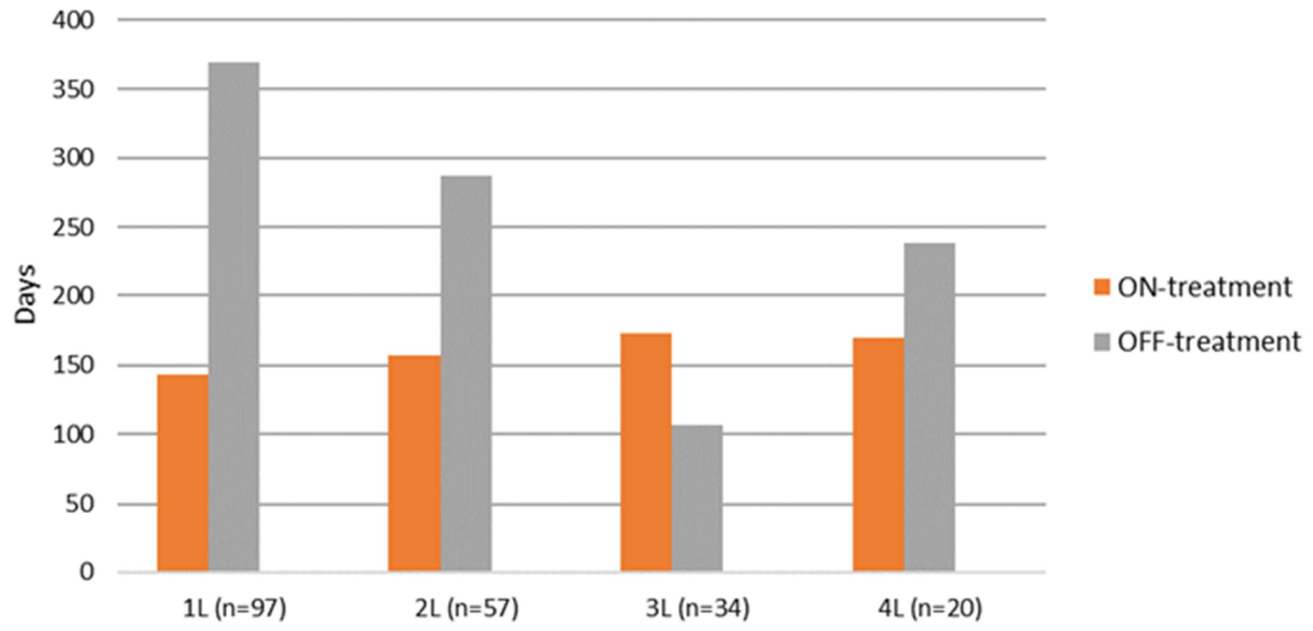

Figure 3. Mean length of the first four treatment lines (1L-4L) of multiple myeloma, including time-on-treatment and time-off-treatment periods, among 97 adults treated for active multiple myeloma at Kymenlaakso Central Hospital, Kymsote, Finland, during 2009-2016. Analysis regarding the off-treatment periods considered all patients whether or not they had a next line treatment during the follow-up.

Table 4. Outcomes associated with the first four treatment lines, among 97 adults treated for active multiple myeloma at Kymenlaakso Central Hospital, Kymsote, Finland, during 2006-2019.

\begin{tabular}{|c|c|c|c|c|}
\hline \multicolumn{5}{|l|}{ Treatment response - no. $(\%)$} \\
\hline & $1 \mathrm{~L}(\mathrm{n}=97,100 \%)$ & $2 \mathrm{~L}(\mathrm{n}=57,100 \%)$ & $3 \mathrm{~L}(\mathrm{n}=34,100 \%)$ & $4 \mathrm{~L}(\mathrm{n}=20,100 \%)$ \\
\hline $\mathrm{CR}$ & $4(4.1)$ & $1(1.8)$ & $0(0.0)$ & $0(0.0)$ \\
\hline VGPR or PR & $68(70.1)$ & $40(70.2)$ & $19(55.9)$ & $10(50.0)$ \\
\hline MR or SD & $19(19.6)$ & $14(24.5)$ & $9(26.5)$ & $6(30.0)$ \\
\hline PD & $6(6.2)$ & $2(3.5)$ & $6(17.6)$ & $4(20.0)$ \\
\hline Received new treatment line & $57(58.8)$ & $34(59.6)$ & $20(58.8)$ & $10(50.0)$ \\
\hline \multicolumn{5}{|c|}{ Reason for stopping the treatment - no. (\%) } \\
\hline & $1 \mathrm{~L}(\mathrm{n}=96,100 \%)$ & $2 \mathrm{~L}(\mathrm{n}=57,100 \%)$ & $3 \mathrm{~L}(\mathrm{n}=33,100 \%)$ & $4 \mathrm{~L}(\mathrm{n}=19,100 \%)$ \\
\hline Good response $^{\mathrm{a}}$ & $48(50.0)$ & $25(43.8)$ & $15(45.4)$ & $8(42.1)$ \\
\hline Lack of response & $19(19.8)$ & $13(22.8)$ & $9(27.3)$ & $1(5.3)$ \\
\hline Disease progression & $9(9.4)$ & $5(8.8)$ & $7(21.2)$ & $6(31.5)$ \\
\hline Adverse drug reaction & $20(20.8)$ & $14(24.6)$ & $2(6.1)$ & $4(21.1)$ \\
\hline
\end{tabular}

a Note that "good response" was provided without a specification. 1L, First Treatment Line; 2L, Second Treatment Line; etc.; CR, Complete Response; MR, Minimal Response; PD, Progressive Disease; PR, Partial Response; SD, Stable Disease; VGPR, Very Good Partial Response. 


\subsection{Treatment Outcomes}

VGPR or PR was the most common outcome across $1 \mathrm{~L}-$ $4 \mathrm{~L}$ but the number of patients with this outcome decreased from $68(70.1 \%)$ in $1 \mathrm{~L}$ to ten $(50.0 \%)$ in $4 \mathrm{~L}$ (Table 4 ). Concomitantly, the relative number of patients with MR or SD increased from 19 patients $(19.6 \%)$ in $1 \mathrm{~L}$ to six patients $(30.0 \%)$ in $4 \mathrm{~L}$, and the relative number of patients with $\mathrm{PD}$ increased from six patients $(6.2 \%)$ in $1 \mathrm{~L}$ to four patients $(20.0 \%)$ in $4 \mathrm{~L}$ (Table 4$)$. Only four patients $(4.1 \%)$ in $1 \mathrm{~L}$ and one patient (1.8\%) in 2L obtained the CR (Table 4).

Across $1 \mathrm{~L}$ to $4 \mathrm{~L}$ treatment, the most common reason for stopping the treatment was obtaining a "good response", although the relative number of these patients slightly decreased along the subsequent treatment lines, from 48 $(50.0 \%)$ in $1 \mathrm{~L}$ to eight $(42.1 \%)$ in $4 \mathrm{~L}$ (Table 4$)$. "Lack of response" and "adverse drug reaction" were the second and third most common reasons for stopping the treatment in $1 \mathrm{~L}(\mathrm{n}=19,19.8 \%$ and $\mathrm{n}=20,20.8 \%$, respectively) and $2 \mathrm{~L}$ $(n=13,22.8 \%$ and $n=14,24.6 \%$, respectively) (Table 4). "Lack of response" further increased in $3 \mathrm{~L}(\mathrm{n}=9,27.3 \%)$ but was a minor reason in $4 \mathrm{~L}(\mathrm{n}=1,5.3 \%)$. The occurrence of "adverse drug reaction" was notably low in $3 \mathrm{~L}(\mathrm{n}=2$, $6.1 \%$ ). "Disease progression" as a reason for stopping the treatment steadily increased from nine patients $(9.9 \%)$ in $1 \mathrm{~L}$ to six patients $(31.5 \%)$ in $4 \mathrm{~L}$ (Table 4$)$. The relative number of patients continuing to the following treatment line remained roughly stable from $1 \mathrm{~L}$ to $4 \mathrm{~L}$ (57\%) (Table 4).

A

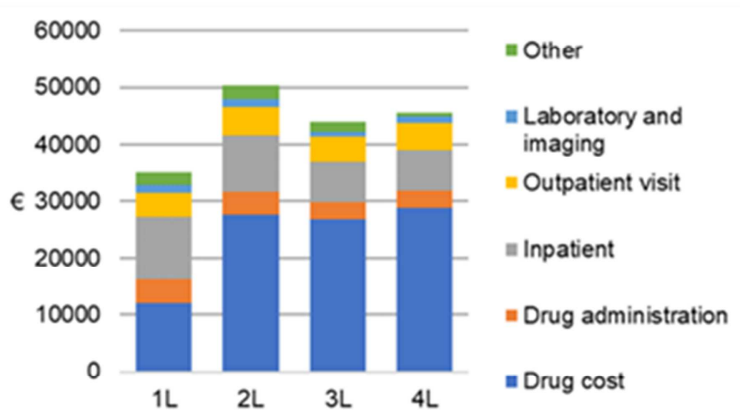

C

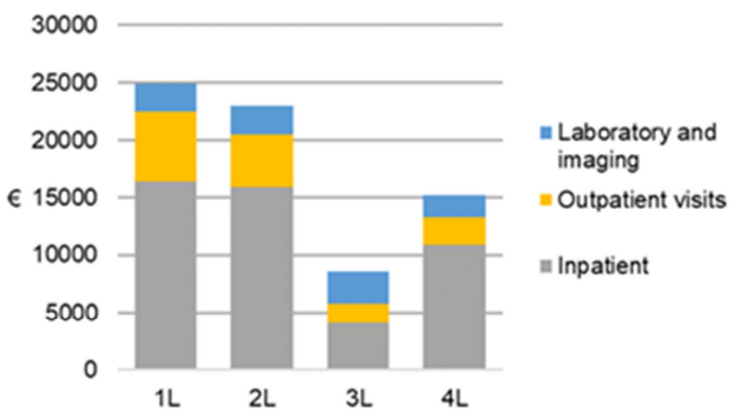

\subsection{Patient Burden and Costs of Travels to Health Care Visits for Oral or Infusion Administration}

Costs associated with HCRU were assessed for each type of expenditure during 1L-4L (Figure 4). Mean per patient HCRU costs associated with the on-treatment period ranged from $35000 €$ (7 $300 €$ per 30 days) in $1 \mathrm{~L}$ to $50400 €$ (9 600 $€$ per 30 days) in 2L (Figure 4). Drug costs represented the greatest proportion of total healthcare costs and increased across $1 \mathrm{~L}-4 \mathrm{~L}$, from $12000 €$ per patient ( $2500 €$ per 30 days) in $1 \mathrm{~L}$ to $28700 €$ per patient (5 $100 €$ per 30 days) in $4 \mathrm{~L}$ (Figure 4). The greatest increase in drug costs per patient was observed between $1 \mathrm{~L}$ and $2 \mathrm{~L}$ and explained most of the difference in total costs related to active treatment between these two treatment lines (Figure 4). The second greatest proportion of costs of on-treatment was due to inpatient costs which ranged from $7000 €(1200 €$ per 30 days $)$ in $3 \mathrm{~L}$ to 11 $100 €(2300 €$ per 30 days) in 1L (Figure 4).

Since the drug costs represented the major expenditure during the active treatment, patient burden and costs related to travels to the treatments stratified by type of drug administration were analysed in more detail. Among the 97 patients included in the study, the mean duration of oral treatments was higher than treatments administered by infusion across $1 \mathrm{~L}-4 \mathrm{~L}$ (173-228 days versus $122-147$ days for $1 \mathrm{~L}-4 \mathrm{~L}$ ) (Table 5). However, the mean number of visits to specialised health care units for oral treatments was lower compared with that observed for infusions (4.0-8.1 visits versus $12.1-15.8$ visits in total) (Table 5).

B

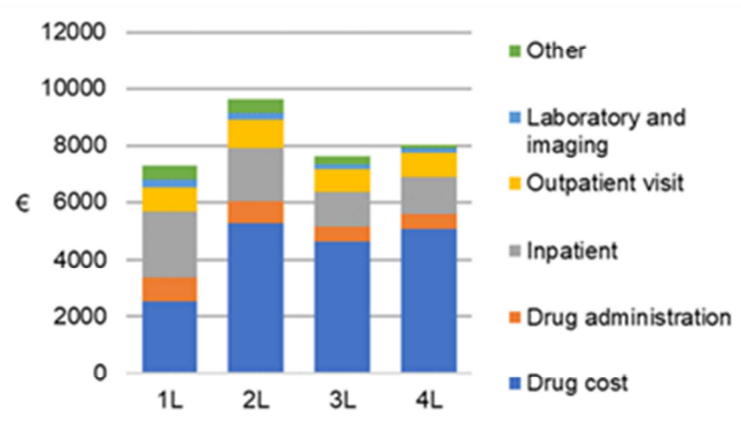

D

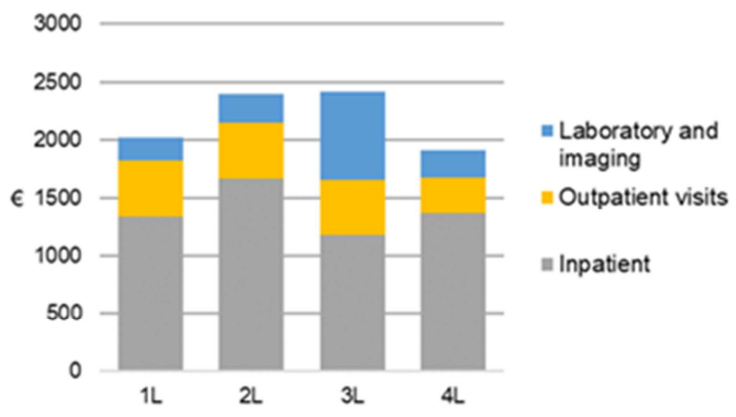

Figure 4. Mean per patient ( $A$ and $C$ ) and per patient per 30 days ( $B$ and D) costs $(\Theta)$ associated with HCRU during on-treatment (A and B) and off-treatment periods $(C$ and $D)$ of the first four treatment lines $(1 L-4 L)$, among 97 adults treated for active multiple myeloma at Kymenlaakso Central Hospital, Kymsote, Finland, during 2009-2016. 
Among the sub-cohort of 63 patients treated at Kymenlaakso Central Hospital during the last two years of the study and whose travels to health care visits were assessed inclusive, mean per patient travel costs related to oral treatments were clearly lower across $1 \mathrm{~L}-4 \mathrm{~L}$ (from $90 €$ in $3 \mathrm{~L}$ to $150 €$ in $1 \mathrm{~L}$, per 30 days of treatment) compared with the respective costs of infusions (from $360 €$ in $4 \mathrm{~L}$ to $460 €$ in $1 \mathrm{~L}$, per 30 days of treatment) (Table 6). Baseline characteristics of the sub-cohort $(n=63)$ where travel costs were determined were found to be comparable to the full cohort.

Table 5. Duration of treatments and the number of specialised health care visits associated with oral versus infusion treatments of the first four treatment lines, among 97 adults treated for active multiple myeloma at Kymenlaakso Central Hospital, Kymsote, Finland, during $2009-2016$.

\begin{tabular}{|c|c|c|c|c|c|c|c|c|}
\hline & \multicolumn{4}{|c|}{ Oral treatment } & \multicolumn{4}{|c|}{ Infusion treatment } \\
\hline & $1 L(n=40)$ & $2 L(n=29)$ & $3 L(n=19)$ & $4 L(n=9)$ & $1 L(n=57)$ & $2 \mathrm{~L}(\mathrm{n}=28)$ & $3 L(n=15)$ & $4 \mathrm{~L}(n=11)$ \\
\hline $\begin{array}{l}\text { Duration of treatment } \\
\text { (days), mean (SD) }\end{array}$ & $\begin{array}{l}174.2 \\
(126.9)\end{array}$ & $\begin{array}{l}173.3 \\
(122.7)\end{array}$ & $\begin{array}{l}194.1 \\
(133.1)\end{array}$ & $\begin{array}{l}228.4 \\
(213.4)\end{array}$ & $123.5(65.5)$ & $\begin{array}{l}140.2 \\
(102.3)\end{array}$ & $146.7(75.9)$ & $122.3(47.2)$ \\
\hline $\begin{array}{l}\text { Number of visits, } \\
\text { mean (SD) }\end{array}$ & $5.5(8.3)$ & $8.0(9.7)$ & $4.0(3.4)$ & $8.1(10.8)$ & $14.5(12.0)$ & $15.8(16.1)$ & $12.1(13.0)$ & $13.7(10.1)$ \\
\hline $\begin{array}{l}\text { Number of visits per } 30 \\
\text { days, mean (SD) }\end{array}$ & $0.8(1.0)$ & $1.5(1.7)$ & $0.7(0.6)$ & $1.0(0.9)$ & $3.6(2.6)$ & $3.5(3.0)$ & $3.4(3.9)$ & $3.6(2.4)$ \\
\hline
\end{tabular}

1L, First Treatment Line; 2L, Second Treatment Line; etc.

Table 6. Travel costs associated with the specialised health care visits during the first four treatment lines, among a sub-cohort of 63 adults treated for active multiple myeloma at Kymenlaakso Central Hospital, Kymsote, Finland, during 2015-2016.

\begin{tabular}{|c|c|c|c|c|c|c|c|c|}
\hline & \multicolumn{4}{|c|}{ Oral treatment } & \multicolumn{4}{|c|}{ Infusion treatment } \\
\hline & $1 L(n=40)$ & $2 L(n=29)$ & $3 L(n=19)$ & $4 \mathrm{~L}(\mathrm{n}=9)$ & $1 \mathrm{~L}(\mathrm{n}=57)$ & $2 L(n=28)$ & $3 L(n=15)$ & $4 L(n=11)$ \\
\hline $\begin{array}{l}€ \text { per } 30 \text { days, } \\
\text { mean (SD) }\end{array}$ & $149.6(126.7)$ & $149.2(162.2)$ & $89.1(85.8)$ & $103.21(117.2)$ & $460.1(401.4)$ & $359.3(396.6)$ & $458.7(623.2)$ & $358.4(340.9)$ \\
\hline
\end{tabular}

1L, First Treatment Line; 2L, Second Treatment Line; etc.

Majority of the patients in the sub-cohort were retired $(\mathrm{n}=55,87.3 \%)$ and only few $(\mathrm{n}=2,3.2 \%)$ were reported to work actively. Remaining patients were either on a sickness leave $(n=5,7.9 \%)$ or reported to not work $(n=1,1.6 \%)$. Most of the patients $(\mathrm{n}=52,82.5 \%)$ did not need an escort during the health care visits. Based on those patients who needed an escort and with available data on the status of an escort $(n=11)$, the escort was, in most cases, not working ( $\mathrm{n}=8, \quad 72.7 \%$ versus $\mathrm{n}=3,27.3 \%$ who worked) and represented a family member and not health care personnel $(n=10,90.9 \%$ versus $n=1,9.1 \%)$.

\section{Discussion}

\subsection{Patient Characteristics and Survival}

MM patients at Kymenlaakso Central Hospital, Kymsote had similar characteristics compared to available literature from Finland in terms of incidence (5-7/100 000/year; here $7.1 / 100000 /$ year for all recorded actively treated adult patients) and median age of onset (65-70 years; here 70 years for the cohort) $[6,11-13,22]$. Smoldering myeloma is recorded with the same ICD-10 code as MM (C90.0). However, as the study included only treated patients, it is likely a relatively representative population of MM patients. Among 97 patients included in this study, slightly more patients represented males $(54.6 \%)$, the finding which is in accordance with the respective data of the Finnish Cancer Registry (54.1\%) [6].

In this cohort, only half of the patients were tested for chromosomal abnormalities with the number of patients positive for tested aberrations, translocations and del17 in particular, being somewhat lower than expected. These observations could reflect different versions of treatment guidelines and availability of chromosomal analysis.

The first patients in this study were diagnosed in 2009. Despite lower ASCT rates at this time and significant portion of patients representing R-ISS II and III, the median survival of 68 months demonstrates positive outcomes for patients treated for MM at Kymenlaakso Central Hospital. According to the previously published Finnish Hematology Registry-based study [22], the median survival of MM patients diagnosed in Finland during 2009-2013 and who did not receive ASCT was 25.6 months and 46.2 months among patients who received conventional and novel treatment, respectively. The comparison of different populations is difficult for many reasons, e.g. the relative proportion of transplanted patients and differences in the treatment landscape. The results from Kymenlaakso Central Hospital suggest an even more positive outcome for the first two treatment lines - overall response rate was $72-74 \%$ for the first two treatment lines while it was $50-56 \%$ for the third and fourth lines of therapy. Nevertheless, some patients experienced a lack of response and adverse events.

\subsection{Treatment Patterns}

Characteristics of treatment patterns may also reflect the dynamics of treatment recommendations over time [25] and possibly, changes in the reimbursement policy in Finland. Finnish treatment guidelines were updated in 2012, and again in 2017 and 2019. The treatment characteristics possibly associated with previous reimbursement policy include low simultaneous use of PI and IM across all treatment lines, low 
number of patients treated with lenalidomide in $1 \mathrm{~L}$, and rather short duration of treatments possibly reflecting the recommendation to treat patients "until best response". Currently, simultaneous use of PI and IM is recommended even in $1 \mathrm{~L}$, lenalidomide is also reimbursed in $1 \mathrm{~L}$ if the patient is not eligible for ASCT, and patients are more often treated "until progression". Short treatment durations may partly result from the lack of response and adverse drug reactions further highlighting the need for novel pharmaceuticals, though also costs, reimbursement policy, patient's own decisions, and treatment fatigue may have affected treatment decisions.

In accordance with the RWE from the United States (US), this study indicates that during the last two decades, conventional regimens were used in early treatment lines, while novel regimens were utilised for later therapies [24, 25].

\subsection{Costs Associated with HCRU and Patient Burden}

Substantial economic burden of disease progression has also been reported in previous RWE studies outside of Finland $[15,27]$. In the current study, inpatient costs were already high in $1 \mathrm{~L}$ suggesting that newly diagnosed patients in this study required early, potentially prolonged in-hospital treatments. These findings are supported by a large database study on MM patients diagnosed during 2006-2016 in US [15]. Outpatient treatments are, in fact, preferred in current clinical practice in sparsely populated Finland. This could also reflect the patients' own preferences [31, 32].

The observation that the number of hospital visits and travel costs were clearly lower for oral treatments across the first four treatment lines at Kymenlaakso Central Hospital suggests that oral treatments may reduce patient and hospital resource burden.

\subsection{Limitations}

Limitations of this study include the low number of patients, especially in later treatment lines, and potential biases regarding the identification of individual treatment lines. Although treatments were identified from patient medical charts, a source that provides more reliable data than purely registry-based data set, the identification of individual treatments was yet based on the retrospective assessment of recordings on drug administrations. The retrospective evaluation of the recordings has become more and more challenging due to rapidly changing treatment landscape and clinical practice towards more personalised therapies.

\section{Conclusion}

The results from Kymenlaakso Central Hospital, Kymsote suggest a positive outcome for the first two treatment lines of MM. However, poorer treatment outcomes in later treatment lines and the observation that some patients treated in the first two treatment lines experienced a lack of response and adverse events underline a need for more efficient and better tolerated treatment options across all lines of treatment. The utilisation of novel regimens was low across treatments also at Kymenlaakso Central Hospital further warranting the regular assessment of most recent treatment practices in Finland and abroad.

Drug and secondly, hospital visits represented the major expenditures during the active treatment of MM with drug costs increasing across the treatments. This study demonstrates that oral treatments may indeed reduce patient and hospital resource burden and thus, should be considered in future health economic evaluations of innovative treatments for MM in Finland.

\section{Ethics}

The study was conducted in accordance with the declaration of Helsinki 11 [33].

\section{Funding}

This work was supported by Takeda.

\section{Conflict of Interest}

ST, VV and KT are employed by Takeda (Helsinki, Finland), and MB was employed by Takeda (Helsinki, Finland) at the time of study conduct. TM is employed by Medaffcon Oy (Espoo, Finland). JJ is employed by MedEngine Oy (Helsinki, Finland).

\section{Acknowledgements}

The patients, doctors, and study nurses (Ilona Karvinen and Raisa Pusila) are thanked for making this study possible. Kristiina Uusi-Rauva is acknowledged for providing medical writing services on behalf of Medaffcon Oy (Espoo, Finland).

\section{References}

[1] Röllig C, Knop S, Bornhäuser M. Multiple myeloma. The Lancet. 2015; 385: 2197-2208.

[2] Barwick BG, Gupta VA, Vertino PM, et al. Cell of Origin and Genetic Alterations in the Pathogenesis of Multiple Myeloma. Front Immunol. 2019; 10: 1121.

[3] Rajkumar SV, Dimopoulos MA, Palumbo A, et al. International Myeloma Working Group updated criteria for the diagnosis of multiple myeloma. The Lancet Oncology. 2014; 15: e538-e548.

[4] The National Cancer Institute (NCI). The Surveillance, Epidemiology, and End Results (SEER). [Internet]. The National Cancer Institute. 2020 [cited 2020 Jul 7]. Available from: https://cancer.gov.

[5] ECIS - European Cancer Information System [Internet]. European Union. 2018 [cited 2020 Jul 8]. Available from: https://ecis.jrc.ec.europa.eu. 
[6] The Finnish Cancer Registry. Statistics and Research. [Internet]. The Finnish Cancer Registry. 2020 [cited 2020 Jul 8]. Available from: https://cancerregistry.fi/.

[7] Kolovos S, Nador G, Kishore B, et al. Unplanned admissions for patients with myeloma in the UK: Low frequency but high costs. Journal of Bone Oncology. 2019; 17: 100243.

[8] Scelo G, Li P, Chanudet E, et al. Variability of Sex Disparities in Cancer Incidence over 30 Years: The Striking Case of Kidney Cancer. European Urology Focus. 2018; 4: 586-590.

[9] Cowan AJ, Allen C, Barac A, et al. Global Burden of Multiple Myeloma: A Systematic Analysis for the Global Burden of Disease Study 2016. JAMA Oncol. 2018; 4: 1221.

[10] Rosenberg PS, Barker KA, Anderson WF. Future distribution of multiple myeloma in the United States by sex, age, and race/ethnicity. Blood. 2015; 125: 410-412.

[11] Sinisalo, Marjatta, Laine, Outi. Myelooma [Internet]. Duodecim Terveysportti health portal. 2018 [cited 2020 Feb 26]. Available from: https://www.terveysportti.fi/apps/ltk/ykt00388?toc=23075.

[12] Säily, Marjaana, Silvennoinen, Raija, Jantunen, Esa, et al. Monimuotoinen myelooma. Duodecim. 2019; 135: 11711179.

[13] Putkonen M, Silvennoinen R. Multippeli myelooma ja muut gammapatiat. In: Porkka K, Lassila R, Remes K, Savolainen E-R, editors. Veritaudit. 4th ed. Helsinki: Duodecim 2015; p. 403-434.

[14] Finnish Hematology Association (SHY) [Internet]. Finnish Hematology Association (SHY). 2020 [cited 2020 Jul 6]. Available from: https://www.hematology.fi/fi.

[15] Hagiwara M, Panjabi S, Delea T, et al. Burden of disease progression in patients with multiple myeloma in the US. Leukemia \& Lymphoma. 2019; 1-9.

[16] Kumar SK, Callander NS, Alsina M, et al. NCCN Guidelines Insights: Multiple Myeloma, Version 3.2018. J Natl Compr Canc Netw. 2018; 16: 11-20.

[17] Moreau P, San Miguel J, Sonneveld P, et al. Multiple myeloma: ESMO Clinical Practice Guidelines for diagnosis, treatment and follow-up. Annals of Oncology. 2017; 28: iv52-iv61.

[18] Kumar SK, Rajkumar SV, Dispenzieri A, et al. Improved survival in multiple myeloma and the impact of novel therapies. Blood. 2008; 111: 2516-2520.

[19] Kazandjian D. Multiple myeloma epidemiology and survival: A unique malignancy. Seminars in Oncology. 2016; 43: 676681.

[20] Ludwig H, Beksac M, Blade J, et al. Current Multiple Myeloma Treatment Strategies with Novel Agents: A European Perspective. The Oncologist. 2010; 15: 6-25.

[21] Raju GK, Gurumurthi K, Domike R, et al. A Benefit-Risk Analysis Approach to Capture Regulatory Decision-Making: Multiple Myeloma. Clin Pharmacol Ther. 2018; 103: 67-76.
[22] Remes K, Anttila P, Silvennoinen R, et al. Real-world treatment outcomes in multiple myeloma: Multicenter registry results from Finland 2009-2013. Bertolini F, editor. PLoS ONE. 2018; 13: $\mathrm{e} 0208507$.

[23] Petrakis I, Kontogiorgis C, Nena E, et al. Unraveling innovation potential in the real-world setting: eighteen novel agents with twenty-six approved European indications, in the management of leukemias, lymphomas, and multiple myeloma. Expert Review of Hematology. 2019; 12: 1063-1075.

[24] Jagannath S, Roy A, Kish J, et al. Real-world treatment patterns and associated progression-free survival in relapsed/refractory multiple myeloma among US community oncology practices. Expert Review of Hematology. 2016; 9: 707-717.

[25] Song X, Cong Z, Wilson K. Real-world treatment patterns, comorbidities, and disease-related complications in patients with multiple myeloma in the United States. Current Medical Research and Opinion. 2016; 32: 95-103.

[26] Teitelbaum A, Ba-Mancini A, Huang H, et al. Health Care Costs and Resource Utilization, Including Patient Burden, Associated With Novel-Agent-Based Treatment Versus Other Therapies for Multiple Myeloma: Findings Using Real-World Claims Data. The Oncologist. 2013; 18: 37-45.

[27] Clancy Z, Pandya S, Shrestha S, et al. Real-world economic outcomes of early progression in newly diagnosed multiple myeloma (NDMM) patients (Pts). JCO. 2018; 36: 194-194.

[28] Mankinen P, Vihervaara V, Torvinen S, et al. Costs of administration, travelling, and productivity losses associated with hospital administration of multiple myeloma drugs in Finland. Journal of Medical Econosmics. 2019; 22: 328-335.

[29] Palumbo A, Avet-Loiseau H, Oliva S, et al. Revised International Staging System for Multiple Myeloma: A Report From International Myeloma Working Group. JCO. 2015; 33: $2863-2869$.

[30] Rajkumar SV, Harousseau J-L, Durie B, et al. Consensus recommendations for the uniform reporting of clinical trials: report of the International Myeloma Workshop Consensus Panel 1. Blood. 2011; 117: 4691-4695.

[31] Leleu X, Mateos M-V, Delforge $M$, et al. Assessment of Multiple Myeloma Patient Preferences on Treatment Choices: An International Discrete Choice Study. Blood. 2015; 126: 2086-2086.

[32] Eek D, Krohe M, Mazar I, et al. Patient-reported preferences for oral versus intravenous administration for the treatment of cancer: a review of the literature. Patient Prefer Adherence. 2016; 10: 1609-1621.

[33] WMA - The World Medical Association-WMA Declaration of Helsinki - Ethical Principles for Medical Research Involving Human Subjects [Internet]. The World Medical Association, Inc. 2018 [cited 2019 Oct 18]. Available from: https://www.wma.net/policies-post/wma-declaration-of-helsin ki-ethical-principles-for-medical-research-involving-human-su bjects/. 